\title{
Estratificación hemodinámica mediante ecocardiografía transtorácica en pacientes con infarto agudo de miocardio con segmento ST elevado
}

Jorge Hidalgo Rosales ${ }^{1}$, Marcos Pariona Javier ${ }^{1, a}$, Sebastián Reyes Villanes ${ }^{1}$, David Minchola Guardia ${ }^{1}$, Patricia Ríos Navarro ${ }^{1, b}$, Marcos Jáuregui Contreras ${ }^{1}$, Marco Gutiérrez Garibay ${ }^{1}$, Gustavo Miranda Rivas ${ }^{1}$, Mario Zubiate Talledo ${ }^{1, c}$

\section{RESUMEN}

Objetivo: Evaluar la factibilidad de realizar una estratificación hemodinámica en pacientes con infarto agudo de miocardio con segmento ST elevado, mediante el uso de ecocardiografía transtorácica.

Materiales y métodos: Estudio descriptivo, prospectivo y transversal que incorporó a 30 pacientes en ritmo sinusal con diagnóstico de infarto agudo de miocardio con ST elevado, ingresados a la unidad coronaria del Hospital Edgardo Rebagliati Martins. Se estimó: gasto cardíaco, presión de llenado de ventrículo izquierdo, resistencia vascular sistémica y resistencia vascular pulmonar.

Resultados: La edad promedio fue $67 \pm 10.6$ años, predominio masculino (86.7\%). La estimación del gasto cardiaco y la presión de llenado del ventrículo izquierdo fue posible en el $100 \%$ de los individuos. La resistencia vascular sistémica y resistencia vascular pulmonar pudieron ser estimadas en 29 (96.6\%) y 28 (93.3\%) pacientes, respectivamente. La estratificación hemodinámica fue: estadio I (normal): 18 pacientes (60\%), estadio II (congestivo): 6 pacientes (20\%), estadio III (hipovolemia, compromiso de VD): 5 pacientes (16.7\%) y estadio IV (Choque): 1 paciente (3.3\%).

Conclusiones: Se concluye que es factible realizar la estratificación hemodinámica ecocardiográfica transtorácica en la población estudiada.

Palabras clave: Estratificación hemodinámica; ecocardiografía transtorácica; infarto de miocardio.

\section{Hemodynamic stratification using transthoracic echocardiography in patients with ST segment elevation acute myocardial infarction}

\section{ABSTRACT}

Objective: To evaluate the feasibility of performing a hemodynamic stratification in patients with ST segment elevation acute myocardial infarction using transthoracic echocardiography.

Materials and methods: A descriptive, prospective and cross-sectional study that included 30 patients in sinus rhythm with ST segment elevation acute myocardial infarction, admitted to the coronary care unit of Hospital Edgardo Rebagliati Martins. Cardiac output, left ventricle filling pressure, systemic vascular resistance and pulmonary vascular resistance were estimated.

Results: The mean age was $67 \pm 10.6$ years old, with male predominance $(86.7 \%)$. The estimation of cardiac output and left ventricle filling pressure was possible in $100 \%$ of the individuals. The systemic vascular resistance and pulmonary vascular resistance could be estimated in 29 (96.6\%) and 28 (93.3\%) patients, respectively. The hemodynamic stratification was as follows: stage I (normal): 18 patients $(60 \%)$, stage II (congestive): 6 patients (20\%), stage III (hypovolemia, RV infarction): 5 patients $(16.7 \%)$ and stage IV (shock): 1 patient $(3.3 \%)$.

Conclusions: It is concluded that a transthoracic echocardiographic hemodynamic stratification may be performed in the studied population.

Keywords: Hemodynamic stratification; transthoracic echocardiography; myocardial infarction.

1. Departamento de Cardiología, Hospital Edgardo Rebagliati Martins.

a. Docente de la Universidad de San Martín de Porres.

b. Docente de la Universidad Peruana de Ciencias Aplicadas.

c. Docente de la Universidad Nacional Mayor de San Marcos. 


\section{INTRODUCCIÓN}

Es innegable la importancia de la estratificación hemodinámica para el pronóstico y tratamiento de los pacientes con infarto agudo de miocardio desde su introducción en la práctica clínica por Forrester ${ }^{(1)}$ en 1977. Aunque inicialmente obtenida por técnicas invasivas, el desarrollo de técnicas no invasivas como el ecocardiograma transtorácico, permite la valoración de la hemodinámica del paciente de manera inocua, a bajo costo y en forma reiterativa. El desarrollo de múltiples índices para los diferentes parámetros hemodinámicos en el transcurso del tiempo ha llevado a la publicación de diversas guías para su uso en años recientes ${ }^{(2-4)}$. La ecocardiografía presenta bondades, pero también limitaciones, siendo las principales: la disponibilidad del ecocardiógrafo, la capacitación del personal involucrado en su uso, el tiempo requerido para realizar el examen y la elaboración de esquemas prácticos de ejecución a partir de la información científica disponible.

Existe abundante literatura con respecto al uso de la ecocardiografía en la evaluación hemodinámica no invasiva en diferentes escenarios, especialmente en los pacientes en estado crítico ${ }^{(5-12)}$. Sin embargo, la información en lo referente al caso específico de los pacientes con infarto agudo de miocardio con segmento ST elevado (IAMSTE) es escasa; en tal sentido se diseñó un estudio prospectivo, descriptivo y transversal para determinar la factibilidad de realizar una estratificación hemodinámica mediante ecocardiografía transtorácica tipo Forrester ${ }^{(1)}$, y discutir los aspectos clínicos derivados de esta investigación.

\section{MATERIALES Y MÉTODOS}

De un universo poblacional de 35 pacientes con IAMSTE ingresados a unidad coronaria del Hospital Edgardo Rebagliati Martins, entre diciembre 2013 y marzo 2014, se seleccionaron 30 pacientes. Se excluyeron pacientes en ritmo no sinusal (fibrilación auricular, portadores de marcapaso) y con mala ventana ecocardiográfica. Seis pacientes precisaron ventilación mecánica (cinco con congestión pulmonar clínica y uno con choque por compromiso de cara inferior y ventrículo derecho). Los estudios se realizaron con un ecocardiógrafo Philips HD11XE, con un transductor multifrecuencia de $2-4 \mathrm{MHz}$, a través de los abordajes convencionales.
Se estimaron las siguientes variables hemodinámicas:

1. Estimación del Gasto Cardíaco (GC): Se estimó el $\mathrm{GC}$ por el método descrito por Lewis ( $\mathrm{GC}=0.785 \mathrm{IxIVT}$ tsvixFCXTSVI2) ${ }^{(13)}$.

2. Estimación de la presión de llenado de ventrículo izquierdo (PLVI): Se asumieron las recomendaciones para la estimación de la PLVI vigentes para función cardíaca disminuida $^{(2)}$, usando los índices: E/e', E/A, TDE, E/Vp y TRIV/t(E-e'); no se estudió el flujo de venas pulmonares por dificultad en su visualización en los pacientes en ventilación mecánica.

3. Estimación de la resistencia vascular periférica (RVS): La RVS fue calculada utilizando la fórmula clásica, la presión de atrio derecho se estimó a través del comportamiento de la vena cava inferior ${ }^{(14)}$ y para los pacientes sometidos a ventilación mecánica se utilizó la fórmula que relaciona el flujo de venas supra hepáticas $[\mathrm{PAD}(\mathrm{mmHg})=21.6-24 \mathrm{xFS}]^{(15)}$.

4. Estimación de la resistencia vascular pulmonar (RVP): La RVP fue medida a través del estudio de la relación de la velocidad máxima del flujo regurgitante tricuspídeo con el flujo del tracto de salida del ventrículo derecho, según lo propuesto por Abbas $[R V P(U \text { Wood })=(10 x V t / \text { IVTtsvd })+0.16]^{(16,17)}$.

Los estudios fueron realizados por tres operadores expertos en ecocardiografía.

Los datos se procesaron con el programa estadístico STATA versión 11. Se empleó el test exacto de Fischer para las variables cualitativas y el Test U de Mann Whitney Wilcoxon para las variables cuantitativas, con el fin de determinar si existían diferencias significativas entre los promedios de dos categorías. Se consideró que existía una diferencia significativa cuando $\mathrm{p}<0.05$.

\section{RESULTADOS}

La edad promedio fue $67 \pm 11$ años, el $86.7 \%$ de la población fueron varones y el $97 \%$ fueron mayores de 50 años de edad. La distribución según la localización del infarto fue: cara anterior $(53.3 \%)$, cara inferior $(43.3 \%)$ y cara lateral (3.3\%). En 28 pacientes $(93.3 \%)$ se realizó coronariografía, encontrándose como arteria responsable del infarto: descendente anterior $(46.7 \%)$, coronaria derecha $(35.7 \%)$, circunfleja (3.3\%), descendente posterior $(6.6 \%)$ y sin lesiones significativas (3.3\%).

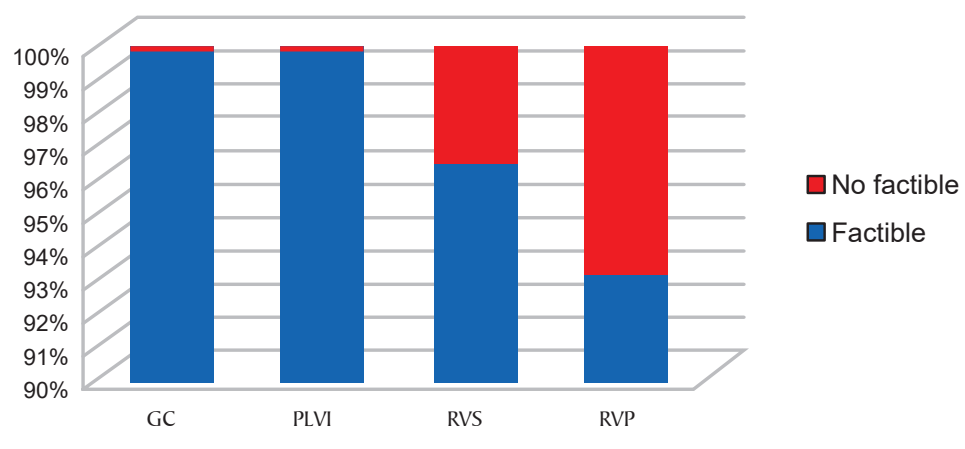

Figura 1. Factibilidad para realizar estratificación hemodinámica 
La figura 1 muestra la factibilidad para estimar los índices necesarios para la estratificación hemodinámica; el GC y PLVI fueron calculados en la totalidad de los casos; la RVS y RVP no se pudieron determinar en todos los pacientes, básicamente por dificultades para estimar la presión de aurícula derecha en un paciente y la velocidad máxima de regurgitación tricuspídea en dos pacientes, todos ellos en ventilación mecánica.

En la figura 2 se muestra la factibilidad de obtener diversos índices de valoración de la PLVI; en todos los pacientes se pudo estimar E/e' y E/A; una factibilidad aceptable se logró para TDE y E/Vp, con dificultades técnicas debido a la fusión de ondas en un individuo taquicárdico y una mala señal en el modo $\mathrm{M}$-color para cada índice respectivamente; finalmente el índice TRIV/t(E-e'), que es resaltado en las guías actuales como un buen indicador ${ }^{(16,18)}$ resultó el más difícil de calcular por su laboriosidad técnica y tuvo la menor factibilidad.

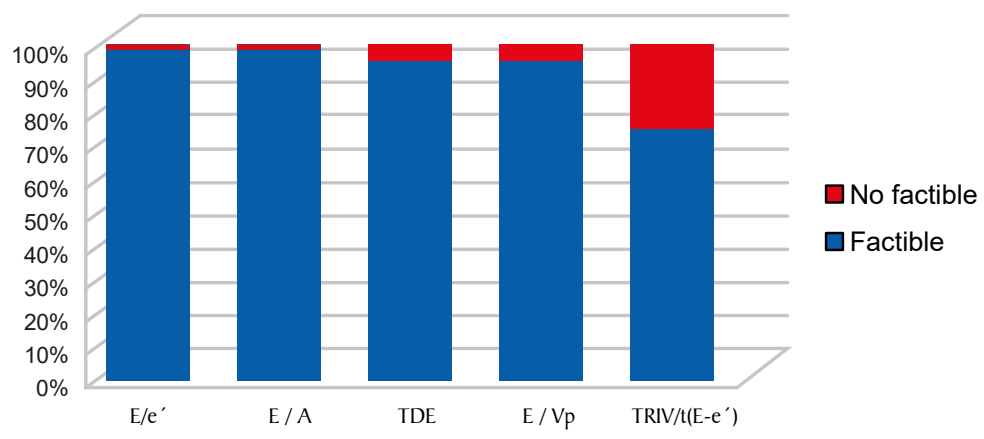

Figura 2. Factibilidad para obtener los índices de PLVI

En la tabla 1 se exponen las características cualitativas de cada indicador asumiendo la relación E/e' como patrón de oro en la estimación de la PLVI, dado que cinco de los siete pacientes estimados con elevación de PLVI por el indicador E/e' estaban en ventilación mecánica por congestión clínico-radiológica. Los indicadores con mayor sensibilidad para determinar elevación de PLVI fueron: E/A>1 y TDE 200 mseg. Los más específicos fueron: $E / A>2$, $\mathrm{E} / \mathrm{Vp}>2$, TDE $<150 \mathrm{~ms}$ y TRIV/t(E-e') $<2$. El coeficiente de regresión simple más destacado fue el de $\mathrm{E} / \mathrm{Vp}$.

Tabla 1. Sensibilidad, especificidad, valor predictivo positivo y valor predictivo negativo de los índices de PLVI

\begin{tabular}{|c|c|c|c|c|c|c|}
\hline Índice & Valor & S (\%) & $E(\%)$ & VPP (\%) & VPN (\%) & $r$ \\
\hline \multirow[t]{3}{*}{ E/A } & $>2$ & 14 & 100 & 100 & 79.3 & 0.35 \\
\hline & $>1.5$ & 57 & 91 & 66.7 & 87.5 & \\
\hline & $>1$ & 71.4 & 65 & 38.5 & 88.2 & \\
\hline \multirow[t]{2}{*}{ TDE } & $<150$ & 42.9 & 77.3 & 38 & 80.9 & 0.21 \\
\hline & $<200$ & 71.4 & 36.4 & 26.3 & 80 & \\
\hline \multirow[t]{2}{*}{$E / V p$} & $>2.5$ & 50 & 95 & 66.6 & 84.6 & 0.56 \\
\hline & $>2$ & 50 & 78.3 & 28.6 & 81.8 & \\
\hline TRIV/t(E-e') & $<2$ & 40 & 78 & 33 & 82.4 & 0.17 \\
\hline
\end{tabular}

E/A: relación entre onda $E$ y A del flujo mitral. TDE: tiempo de desaceleración de onda E mitral. TRIV: tiempo de relajación isovolumétrica. TRIV/t(E-e'): cociente entre el TRVI y la diferencia entre el tiempo entre la onda $\mathrm{R}$ y el inicio de la onda $\mathrm{E}$ y onda e' (promediada medial y lateral). E/Vp: relación entre onda $\mathrm{E}$ mitral y velocidad de propagación del llenado rápido mitral en modo $M$ color. S: sensibilidad. E: especificidad. VPP: valor predictivo positivo. VPN: valor predictivo negativo. $r$ : coeficiente de correlación de variables respecto a E/e'.

La figura 3 muestra los resultados de la estratificación hemodinámica en la población estudiada. 


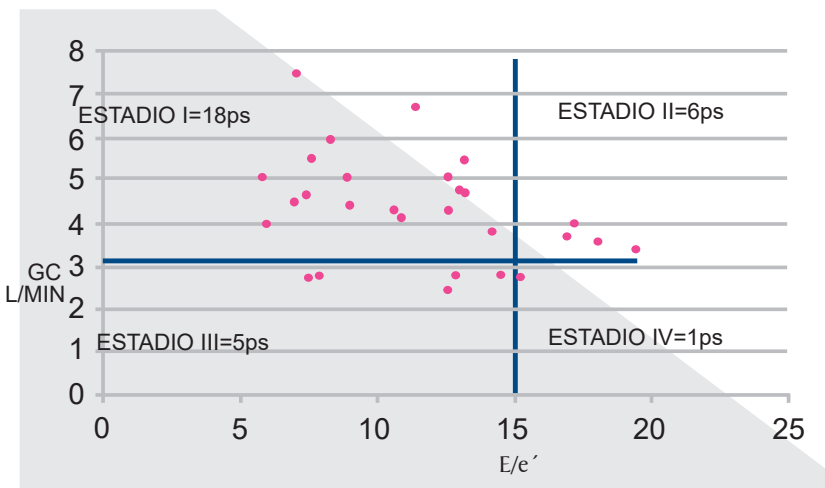

Figura 3. Estratificación hemodinámica

\section{DISCUSIÓN}

Nuestro estudio demuestra que es factible realizar una estratificación hemodinámica con ecocardiografía transtorácica en pacientes con IAMSTE, tal hallazgo es coherente con los reportes de la literatura ${ }^{(19,20)}$. Una limitación en nuestro estudio fue la latencia prolongada entre el inicio del dolor torácico hasta la realización del ecocardiograma debido a la falta de ecocardiógrafo en emergencia.

En cuanto al GC, fue posible estimarlo por el método descrito; creemos que la medición del GC por ETT tiene la ventaja de aplicarse directamente en cavidades izquierdas en comparación con otros métodos (termodilución, Fick o dilución de indicadores).

En cuanto a la estimación de la PLVI que deviene en la parte más compleja de la evaluación no invasiva, hemos utilizado los índices recomendados para individuos con depresión de la función sistólica ventricular izquierda; nuestro indicador cardinal fue la relación $E / e^{\prime}>15^{(2,21,22}$ el cual fue refrendado con el cuadro clínico- radiológico.

Adicionalmente el patrón doppler transmitral E/A, resultó de gran utilidad en nuestra estimación; de tal manera que un patrón pseudonormal o restrictivo, al igual que un TDE $<200$ mseg mostraron aceptables niveles de sensibilidad y especificidad para evocar y reforzar una elevación de la $\mathrm{PLVI}$ mostrada por una relación $\mathrm{E} / \mathrm{e}^{\prime}$ incrementada.

El TRIV/t(E-e') ${ }^{(2,15)}$ fue un índice poco eficiente, debido en parte a la laboriosidad que requería su medición y por otro lado la menor sensibilidad mostrada, sin embargo la presencia de un TRIV/t(E-e') < 2 se mostró bastante específica para expresar la elevación de PLVI. E/Vp fue el único índice que mostró una correlación aceptable con la relación $\mathrm{E} / \mathrm{e}^{\prime}$.

Nosotros proponemos la estimación de la PLVI multíndice; centrándonos en la relación $\mathrm{E} / \mathrm{e}^{\prime}$, refrendado por un flujo transmitral pseudonormal o mejor aún restrictivo. Adicionalmente puede afianzarse la estimación con $\mathrm{E} / \mathrm{Vp}$ y TRIV/t(E-e').
La RVS también tuvo una aceptable factibilidad para ser estimada, la limitación más importante fue la estimación de la presión de aurícula derecha, sea por una mala ventana subcostal o por permanencia en ventilación mecánica. Algunos estudios ${ }^{(22)}$ han mostrado que un importante porcentaje de los pacientes con choque cardiogénico en IAMSTE son consecuencia de la aplicación de una terapéutica empírica rígida; consideramos que conocer los valores de las resistencias, ayudará a individualizar el tratamiento farmacológico especialmente cuando se utilizan medicamentos vaso activos. La RVP también tuvo una aceptable factibilidad en su estimación; en algunos estudios realizados en población con patología crónica ${ }^{(19,20)}$ se observó una menor factibilidad en su obtención; una de las razones para esta discrepancia podría ser el hecho que nuestros casos con mala visualización del flujo tricuspídeo fueron realzados con solución salina agitada (3 pacientes) ${ }^{(17)}$.

En conclusión, la Estratificación Hemodinámica Ecocardiográfica Transtorácica (EHET) es factible de realizar en pacientes con IAMSTE. La evaluación del gasto cardíaco se mostró fácil y factible; respecto a la estimación multíndice de la presión de llenado de ventrículo izquierdo en individuos con IAMSTE sugerimos que debe tener como eje principal ecocardiográfico la estimación de la relación E/e' y adicionalmente se debe comparar con el estudio del flujo transmitral y la relación $\mathrm{E} / \mathrm{Vp}$. El índice TRIV/t(E-e') resultó muy poco sensible pero altamente específico.

\section{REFERENCIAS BIBLIOGRÁFICAS}

1. Forrester JS, Diamond G, Swan HJ. Correlative Classification of clinical and hemodynamic Function After Acute Myocardial Infarction. Am J Cardiol. 1977;39(2):137-45.

2. Nagueh SF, Appleton CP, Gillebert TC, Marino PN, Oh JK, Smiseth OA, et al. Recommendations for the Evaluation of Left Ventricular Diastolic Function by Echocardiography. J Am Soc Echocardiogr. 2009;22(2):107-33.

3. Porter TR, Shillcutt SK, Adams MS, Desjardins G, Glas KE, Olson JJ, et al. Guidelines for the Use of Echocardiography as a Monitor for Therapeutic Intervention in Adults: A Report 
from the American Society of Echocardiography. J Am Soc Echocardiogr. 2015;28(1):40-56.

4. Nagueh SF, Smiseth OA, Appleton CP, Byrd BF, Dokainish H, Edvardsen $\mathrm{T}$, et al. Recommendations for the Evaluation of Left Ventricular Diastolic Function by Echocardiography: An Update from the American Society of Echocardiography and the European Association of Cardiovascular Imaging. J Am Soc Echocardiogr 2016;29(4):277-314

5. Memtsoudis SG, Rosenberger $P$, Loffler $M$, Eltzschig HK, Mizuguchi A, Shernan SK, et al. The usefulness of transesophageal echocardiography during intraoperative cardiac arrest in non-cardiac surgery. Anesth Analg. 2006;102(6):1653-7.

6. Ritzema JL, Richards AM, Crozier IG, Frampton CF, Melton IC, Doughty RN, et al. Serial Doppler echocardiography and tissue Doppler imaging in the detection of elevated directly measured left atrial pressure in ambulant subjects with chronic heart failure. J Am Coll Cardiol Img 2011;4(9):927-34.

7. Lahm T, McCaslin CA, Wozniak TC, Ghumman W, Fadl YY, Obeidat OS, et al. Medical and surgical treatment of acute right ventricular failure. J Am Coll Card. 2010;56(18):1435-46.

8. Rydman R, Larsen F, Caidahl K, Alam M. Right ventricular function in patients with pulmonary embolism: early and late findings using Doppler tissue imaging. J Am Soc Echocardiogr. 2010;23(5):531-7.

9. Kohli-Seth R, Neuman T, Sinha R, Bassily-Marcus A. Use of echocardiography and modalities of patient monitoring of trauma patients. Curr Opin Anaesth. 2010;23(2):239-45.

10. Torres $C$. Evaluación de método ecocardiográfico para calcular presión diastólica final de ventrículo izquierdo en pacientes con enfermedad coronaria. Revista Peruana de Cardiogia. 2002;28(1):42-9.

11. Jáuregui $M$. Valor pronóstico de la velocidad de propagación del llenado ventricular en pacientes con infarto de miocardio con elevación del segmento ST. Revista Peruana de Cardiología. 2006;32(1):44-9.

12. Ríos JJ, Romero R. Determinación de la presión de llenado ventricular izquierdo por doppler tisular en pacientes con disfunción cardiaca sistólica y diastólica. Revista Peruana de Cardiología. 2007;33(1):16-9.

13. Lewis JF, Kuo LC, Nelson JG, Limacher MC, Quiñones MA. Pulsed Doppler Echocardiographic Determination of Stroke Volume and Cardiac Output: Clinical Validation of two new methods using the apical window. Circulation 1984;70(3):425-31.

14. Kircher BJ, Himelman RB, Schiller N.B. Noninvasive estimation of right atrial pressure from the inspiratory collapse of the inferior vena cava. Am J Cardiol. 1990;66(4):493-6.
15. Nagueh S. Kopelen H. Zoghbi W. Relation of mean right atrial pressure to echocardiographic and Doppler parameters of right atrial and right ventricular function. Circulation. 1996; 93(6):1160-1169

16. Abbas AE, Fortuin FD, Schiller NB, Appleton CP, Moreno CA, Lester SJ. A simple method for noninvasive estimation of pulmonary vascular resistance. J Am Coll Cardiol 2003; 41(6):1021-7.

17. Himelman RB, Stulbarg M, Kircher B, Lee E, kee L, Dean N, et al. Noninvasive evaluation of pulmonary artery pressure during exercise by saline-enhanced doppler echocardiography in chronic pulmonary disease. Circulation. 1989;79(4):863-71.

18. Nagueh SF. Tissue doppler imaging for the assessment of left ventricular diastolic function. J Cardiovasc Ultrasound 2008;16(3):76-9.

19. Florio L, Vignolo G, Centurión R, Manfredi A, Cuña E. Factibilidad de la valoración hemodinámica ecocardiográfica no invasiva en la insuficiencia cardíaca crónica. Rev Ur Cardiol. 2006;21(2):117-23.

20. Pérez Cabrera D, Herrera A, Gómez García. Variables hemodinámicas para la valoración incruenta de la hemodinámica pulmonar. Corsalud. 2012;4(3).215-7.

21. Lester S, Tajik AJ, Nishimura RA, Oh J, Khandheria B, Seward J. Unlocking of diastolic function: Deciphering the Rosetta Stone 10 years later. J Am Coll Cardiol. 2008;51(7):679-89.

22. Hochman JS, Buller CE, Sleeper LA, Boland J, Dvazik V, Sanborn T, et al. Cardiogenic shock complicating acute myocardial infarction-etiologies, management and outcome: a report from the SHOCK trial Registry. J Am Coll Cardiol. 2000;36(3):1063-70.

Fuentes de financiamiento:

Este artículo ha sido financiado por los autores.

\section{Conflictos de interés:}

Los autores declaran no tener ningún conflicto de interés.

\section{Correspondencia:}

Marcos Pariona

Dirección: Libertad 1474. Dpto.401B. Magdalena del Mar

Teléfono: 949209438 / 2613709

Correo electrónico: marcospariona@gmail.com

Recibido: 27 de marzo de 2017 Aprobado: 05 de mayo de 2017 